\title{
A mitochondrial DNA SNP multiplex assigning Caucasians into 36 haplo- and subhaplogroups
}

Mikkelsen, Martin; Rockenbauer, Eszter; Sørensen, Erik; Rasmussen, Michael; Børsting, Claus; Morling, Niels

Publication date:

2008

Document version

Early version, also known as pre-print

Citation for published version (APA):

Mikkelsen, M., Rockenbauer, E., Sørensen, E., Rasmussen, M., Børsting, C., \& Morling, N. (2008). A mitochondrial DNA SNP multiplex assigning Caucasians into 36 haplo- and subhaplogroups. Poster session presented at 22nd International ISFG Congress, København, Denmark. 

assigning Caucasians into 36 haplo- and subhaplogroups Martin Mikkelsen, Eszter Rockenbauer, Erik Sørensen, Michael Rasmussen, Claus Børsting and Niels Morling University of Copenhagen, 11 Frederik V's Vej, DK-2100 Copenhagen, Denmark

\section{INTRODUCTION}

Mitochondrial DNA (mtDNA) is a valuable tool for human identification when only low quality and/or limited amounts of nuclear DNA are available. Today, mtDNA analyses are mainly performed by sequencing of the hypervariable regions 1 and 2 (HV1/HV2) within the non-coding control region. The hypervariable regions provide a limited power of discrimination when it comes to human identification. Further discrimination can be achieved by focusing on the entire mitochondrial genome instead of only the control region. DNA sequencing of the entire mtDNA is expensive, tedious and time consuming. The latest developments in mtDNA investigation turns towards more rapid screening methods such as SNP typing. The Single Base Extension (SBE) reaction provides a fast and efficient method for typing SNPs. Several previous studies have linked mtDNA haplogroups to various combinations of SNPs inside the coding region of the mtDNA [1-3]. Here, we present a combination of a multiplex PCR reaction followed by two SBE multiplex reactions typing 33 SNPs with which we can define a total of 36 common Western Eurasian mtDNA haplo- and subhaplogroups.

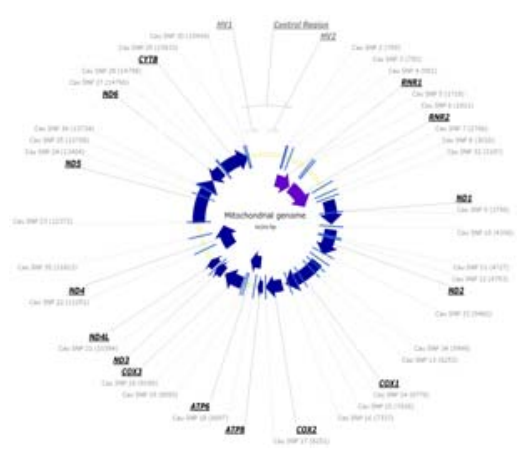

Figure 1: Position of the selected SNPs in the mitochondrial genome. Protein coding genes and rRNA genes are shown (abbreviated).
2A

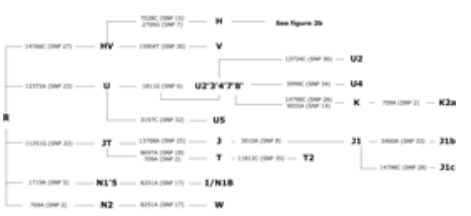

2B

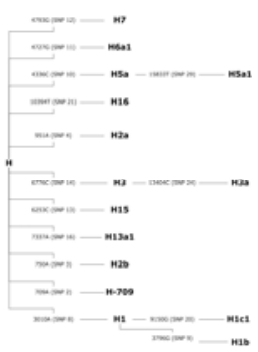

Figure 2: Phylogenic tree of the 36 haplogroups. The position of each SNP and the variation from rCRS are given. (A) Western Eurasian haplogroups typed in the assay $[1,3]$. (B) Haplogroup H's subhaplogroups typed in the assay. [2]

\section{MATERIALS AND METHODS}

A total of 50 samples with known HV1 and HV2 sequences [4] were analyzed. A total of 33 previously described [1-3] SNPs were amplified on 26 amplicons in one multiplex PCR reaction. The 33 SNPs were typed using two separate SBE reactions, a 16-plex and a 17-plex reaction, using the SNaPShot ${ }^{\circledR}$ kit from Applied Biosystems. The SBE reactions were analyzed by capillary electrophoresis using a double injection protocol that allows for two SBE reactions to be analyzed together (Figure 3). The results were analyzed using GeneScan ${ }^{\circledR}$ and Genotyper ${ }^{\circledR}$ softwares. The results were compared to earlier haplotype assignment based on HV1 and HV2 sequencing (Table 1).
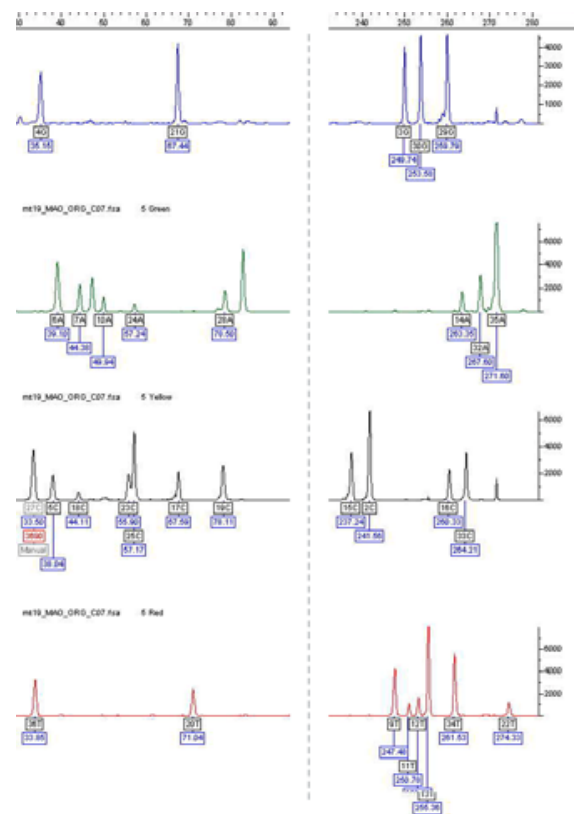

Figure 3: Electropherogram of mtDNA SNP typing. The double injection protocol allows for analysis of the results from the 16- and 17- SBE plexes in the same Genotyper ${ }^{\circledR}$ window.

\section{RESULTS AND DISCUSSION}
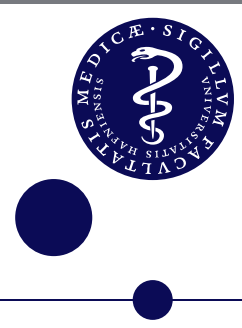

Table 1 shows the mtDNA haplogroup assignments obtained with sequencing and SNP typing. A total of 12 samples were assigned to the exact same haplogroups by the two methods. Eleven samples were typed as haplogroup $\mathrm{H}$ by sequencing, but were further divided into subhaplogroups using the SNP typing method. The results from one sample (mt38) showed inconsistency between the haplogroup assignment obtained with sequencing and SNP typing, and three samples ( $\mathrm{mt14}, \mathrm{mt19}, \mathrm{mt} 40$ ) placed in a $\mathrm{H}$ subhaplogroup by sequencing were placed in another $\mathrm{H}$ subgroup with the SNP typing method. Except for mt38 and two samples that could not be typed, all samples fell into the same branches of the phylogenic tree, when the results from sequencing and SNP typing were compared. Overall, the 48 samples typed in this work were assigned to 20 different haplogroups using the SNP typing method. In comparison, the same 48 samples were assigned to only 16 different haplogroups by sequencing. Our results demonstrate that typing of the 33 coding region SNPs in the mitochondrial genome is a fast and effective method for assigning the major mtDNA haplogroups to individuals.

\section{REFERENCES}

1. Brandstätter A et al. Int.J.Legal Med. 117:291-298, 2003

2. Brandstätter A et al. Electrophoresis 27:2541-2550, 2006

3. Herrnstadt $C$ et al. Am.J.Hum.Genet. 70:1152-1171, 2002

4. Rasmussen EM et al. Progress in Forensic Genetics 10. 1261: 401-3, 2004. 